UVX 2010 (2011) 91-97

DOI: $10.1051 / \mathrm{uvx} / 2011013$

(C) Owned by the authors, published by EDP Sciences, 2011

\title{
Caractérisation spectrale des lasers XUV
}

\author{
A. Klisnick ${ }^{1}$, O. Guilbaud ${ }^{2,6}$, J.P. Goddet ${ }^{3}$, F. Tissandier ${ }^{3}$, L.M. Meng ${ }^{1}$, \\ L. Urbanski ${ }^{4}$, J. Gautier ${ }^{3}$, S. de Rossi ${ }^{5}$, Y. Wang ${ }^{4}$, D. Alessi ${ }^{4}$, D. Martz ${ }^{4}$, \\ B. Luther ${ }^{4}$, G. Maynard ${ }^{6}$, D. Benredjem ${ }^{7}$, A. Calisti ${ }^{8}$, S. Sebban ${ }^{3}$, \\ J.J. Rocca ${ }^{4}$, M. Marconi ${ }^{4}$ et D. Joyeux ${ }^{5}$ \\ 1 ISMO, Bât. 350, CNRS, Université Paris-Sud 11, Orsay, France \\ 2 LASERIX, Université Paris-Sud 11, Orsay, France \\ ${ }^{3}$ LOA, ENSTA-École Polytechnique, Palaiseau, France \\ ${ }^{4}$ NSF Center for EUV Science and Technology Colorado State University, \\ Fort Collins, USA \\ ${ }^{5}$ Lab Charles Fabry Institut d'Optique, Palaiseau, France \\ ${ }^{6}$ LPGP, CNRS, Université Paris-Sud 11, Orsay, France \\ ${ }^{7}$ LAC, CNRS, Université Paris-Sud, Orsay, France \\ ${ }^{8}$ PIIM, CNRS, Univ. de Provence, Marseille, France
}

\begin{abstract}
Résumé. We review recent experimental work devoted to the characterization of the spectral linewidth of three types of XUV lasers pumped by collisional excitation, and operated in either seeded or ASE modes. The spectral properties were deduced from the measurement of the temporal coherence of the XUV laser pulse, using a variable pathlength interferometer.
\end{abstract}

\section{INTRODUCTION, MOTIVATIONS}

Les lasers XUV générés dans des plasmas chauds sont des sources intenses, caractérisées par une émission extrêmement monochromatique. La mesure de leur largeur spectrale, typiquement $\Delta \lambda \mathrm{v} \lambda$ $\sim 10^{-5}$, représente un challenge expérimental car elle se situe à la limite de résolution des spectromètres les plus performants dans ce domaine. Pourtant la connaissance de ces propriétés spectrales est un enjeu important dans la compréhension et l'amélioration de ces sources. En effet, de nombreux processus contribuent de manière complexe à l'élargissement spectral des raies laser XUV (collisions, effet Doppler, transfert radiatif, etc) qui dépendent eux-mêmes étroitement des conditions de densité et de température régnant dans le plasma amplificateur. La prise en compte de ces effets nécessite le recours à des modèles théoriques plus ou moins sophistiqués et surtout à des codes de simulation numériques, en général couplés à des codes hydrodynamique ou de physique atomique. Il est donc important de pouvoir tester la validité de la prédiction de ces codes par une comparaison avec des mesures expérimentales.

Par ailleurs, la largeur spectrale des lasers XUV conditionne plusieurs propriétés essentielles pour leur utilisation à des fins d'applications. Ainsi le taux de photoabsorption de la raie laser X par la matière dépend de largeur spectrale dans laquelle est contenue l'énergie absorbée. D'autre part, d'après le théorème de Wiener-Khintchine, la cohérence temporelle de la source est inversement proportionnelle à sa largeur spectrale. On verra que c'est sur cette propriété que reposent les mesures que nous présentons dans cet article. Enfin, et c'est l'une des principales motivations de notre travail, la largeur spectrale des lasers XUV, typiquement de l'ordre de $8.10^{11} \mathrm{~Hz}$, constitue actuellement une limite pour étendre la

This is an Open Access article distributed under the terms of the Creative Commons Attribution-Noncommercial License 3.0, which permits unrestricted use, distribution, and reproduction in any noncommercial medium, provided the original work is properly cited. 
durée de ces sources dans le domaine sub-picoseconde, ou femtoseconde. Ainsi la durée la plus courte mesurée jusqu'à présent pour un laser XUV émis à 18,9 nm est de 1 picoseconde [1].

Pour atteindre le domaine femtoseconde, il est donc nécessaire d'élargir la bande spectrale des lasers XUV, en jouant si possible sur la contribution des mécanismes d'élargissement. Le but du travail que nous présentons est donc de caractériser la largeur spectrale de différents types de lasers XUV actuellement opérationnels, d'identifier les mécanismes d'élargissement dominants en étudiant le rôle des différents paramètres plasma (densité, températures électronique et ionique), et finalement de proposer des stratégies pour obtenir une largeur spectrale compatible avec une amplification femtoseconde.

Dans cet article nous décrivons d'abord la méthode expérimentale que nous avons utilisée pour mesurer la largeur spectrale extrêmement fine des lasers XUV. Dans la section 3, nous décrivons les résultats que nous avons obtenus pour trois types de lasers XUV, générés par le même mécanisme de pompage, mais par des méthodes expérimentales et pour des conditions de plasma différentes. Les expériences ont été réalisées d'une part au LOA (ENSTA, Palaiseau), d'autre part au Colorado State University (Fort Collins, USA), en collaboration avec les équipes qui développent respectivement ces sources. Enfin le bilan de ces mesures et les pistes que nous envisageons d'explorer seront discutés dans la section 5.

\section{MÉTHODE EXPÉRIMENTALE}

Comme expliqué dans l'introduction, il n'est pas possible, compte tenu de la résolution spectrale des spectromètres XUV actuellement disponibles, de mesurer directement la largeur spectrale des lasers XUV. Nous avons donc recours à la mesure de la cohérence temporelle de la source, qui est liée à la largeur spectrale à travers le théorème de Wiener-Khintchine. Ce théorème stipule que la densité spectrale de puissance d'un signal est la transformée de Fourier de la fonction d'autocorrélation correspondante. Par conséquent la largeur spectrale $\Delta v$ et le temps de cohérence $\tau_{\mathrm{C}}$ sont liés par la relation : $\Delta v=\alpha / \tau_{\mathrm{C}}$, où $\alpha$ est un facteur numérique qui dépend de la forme du profil spectral.

Dans nos expériences le temps de cohérence est mesuré à l'aide d'un interféromètre à division de front d'onde et différence de marche variable, spécifiquement conçu au LCFIO pour ces mesures [2]. La figure 1 montre le principe de fonctionnement de cet interféromètre, placé à environ 3 mètres de la source. Le faisceau laser XUV qu'on cherche à étudier est réfléchi sous incidence rasante $\left(6^{\circ}\right)$ par une paire de dièdres qui forment entre eux un petit angle de quelques milliradians. Après réflexion le faisceau est donc partagé en deux demi-faisceaux qui convergent faiblement l'un vers l'autre. Les franges d'interférence qui se forment dans la zone de recouvrement sont détectées à l'aide d'une CCD XUV. Le plan de la CCD est incliné de $35^{\circ}$ par rapport à la normale, afin d'augmenter l'interfrange apparente sur le détecteur jusqu'à environ 10 pixels. La visibilité des franges, pour une différence de marche nulle entre les deux demi-faisceaux, est donnée par la cohérence spatiale de la source, elle même contrôlée par sa dimension spatiale. La mesure de cohérence temporelle consiste à faire varier la différence de marche entre les deux bras de l'interféromètre, en translatant verticalement un des deux bi-dièdres par rapport à l'autre. La géométrie en bi-dièdre, choisie à la place de simples miroirs, permet de conserver la même géométrie de recouvrement des deux demi-faisceaux, lorsque la différence de marche est augmentée. Ainsi on s'assure que la perte de visibilité des franges observée est bien due à la cohérence temporelle finie de la source, et non à des effets de cohérence spatiale.

Pour des conditions de génération de la source données, on enregistre une série d'interférogrammes pour des valeurs croissantes de la différence de marche. Pour tenir compte des inévitables fluctuations tir à tir, 5 à 10 interférogrammes sont effectués pour chaque différence de marche. La figure 1 montre également un exemple typique d'interférogramme obtenu. Chaque interférogramme est ensuite traité numériquement pour mesurer la visibilité des franges, en utilisant une méthode de transformée de Fourier à fenêtre glissante sur la zone d'interférences [3]. On trace alors la visibilité des franges en fonction de la différence de marche, qui est la fonction d'autocorrélation du signal. Le temps de 

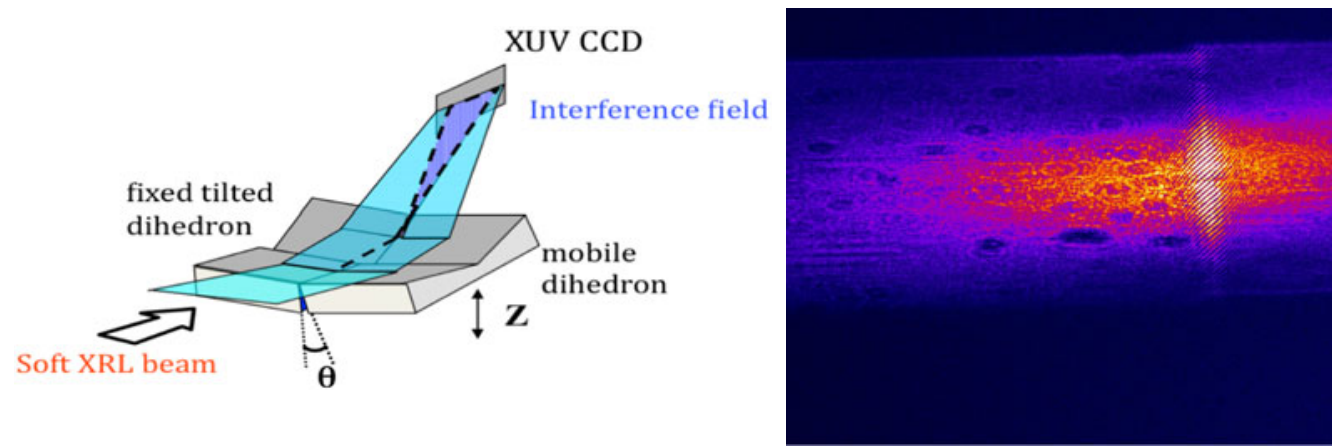

Figure 1. Principe de fonctionnement de l'interféromètre à différence de marche variable. A droite, Exemple d'interférogramme obtenu. Les franges d'interférence apparaissent dans la région de recouvrement des deux demifaisceaux après réflexion sur les miroirs de l'interféromètre.

cohérence $\tau_{\mathrm{C}}$ est défini comme la différence de marche (ddm, mesurée en unité de temps) pour laquelle la visibilité est divisée par e, par rapport à la visibilité maximale (i.e. ddm =0).

\section{3. ÉTUDE COMPARATIVE DE TROIS LASERS XUV COLLISIONNELS}

Les mesures de cohérence temporelle, avec l'interféromètre décrit plus haut, ont été initiées au LULI en 2004 [4], puis poursuivies sur d'autres installations générant différents types de laser XUV [5,6]. Dans cet article, nous résumons les résultats des expériences les plus récentes, dans lesquelles nous avons étudié trois types de laser XUV possédant des caractéristiques différentes. Ces trois lasers XUV ont en commun d'être générés par le même mécanisme de pompage, l'excitation collisionnelle d'ions nickeloïdes et néonoïdes, mais par des méthodes expérimentales et des modes d'amplification notablement différents.

En particulier nous avons pu comparer les deux seuls lasers XUV fonctionnant actuellement en mode injecté par du rayonnement harmonique. Ce nouveau mode d'amplification du laser XUV permet d'améliorer considérablement la qualité optique du faisceau laser XUV, en particulier sa cohérence spatiale, par rapport au mode d'amplification d'émission spontanée (ASE) utilisé jusqu'à récemment. L'injection d'harmonique a d'abord été démontrée au LOA [7], dans un laser XUV pompé par ionisation par champ laser (OFI: Optical Field Ionization) d'un gaz de xénon, puis de krypton [8]. Cette méthode a ensuite été étendue à un laser $X$ transitoire fonctionnant avec du titane [9], puis des éléments plus lourds (jusqu'au cadmium [10]), par l'équipe de JJ Rocca (CSU, Etats-Unis). Du point de vue des propriétés spectrales qui nous intéressent ici, le laser XUV transitoire (injecté ou non) diffère du laser XUV OFI par les paramètres plasma qui règnent dans la zone de gain. En particulier, le pompage transitoire fonctionne à une densité électronique environ 10 fois plus élevée que le pompage OFI, qui repose sur l'ionisation d'un gaz.

Enfin, pour compléter l'étude, nous avons étudié le laser XUV, également développé au CSU, pompé non pas par un laser intense, mais par une décharge électrique rapide dans un plasma d'argon. Là encore l'émission laser est obtenue par pompage par excitation collisionnelle, mais les caractéristiques du plasma amplificateur et du faisceau laser XUV sont très différentes des précédentes. En particulier, la densité électronique est plus faible que pour le pompage transitoire, mais aussi la température ionique est relativement élevée, ce qui favorise l'élargissement Doppler.

Nous présentons les principaux résultats obtenus pour chacun de ces lasers XUV. On verra que ces résultats reflètent bien, qualitativement et quantitativement, les différentes conditions de plasma qui leur correspondent. 


\subsection{Laser XUV OFI en mode injecté}

Nous avons mesuré la cohérence temporelle du laser XUV OFI en mode injecté, développé au LOA [8]. Ce laser est émis à $32 \mathrm{~nm}$ à la longueur d'onde de la raie $4 \mathrm{~d}-4 \mathrm{p}$ du krypton nickeloïde. Dans une première expérience réalisée avec ce laser, nous avions mesuré un temps de cohérence de $\sim 5 \mathrm{ps,} \mathrm{très}$ proche de la durée d'impulsion de cette source, mesurée indirectement [6]. Une nouvelle mesure a été effectuée récemment en améliorant la qualité de l'alignement et des interférogrammes. Ceci nous a permis d'étudier plus en détail l'influence de plusieurs paramètres [11].

En particulier nous avons comparé le temps de cohérence du laser à $32 \mathrm{~nm}$ fonctionnant en mode injecté à celle obtenue en mode ASE (i.e. sans injection d'harmonique). Dans les deux cas la longueur du plasma amplificateur était de $6 \mathrm{~mm}$. Les courbes de visibilité obtenues sont présentées dans la figure 2. Chaque point est la moyenne de 5 à 10 tirs effectués dans les mêmes conditions et la barre d'erreur représente la déviation standard des mesures. Les courbes en traits pleins et pointillés sont les fits des données, correspondant au produit d'une Gaussienne et d'une exponentielle décroissante. Une telle fonction correspond dans l'espace des fréquences spectrales à une convolution d'une Gaussienne et d'une Lorentzienne, ou profil de Voigt.

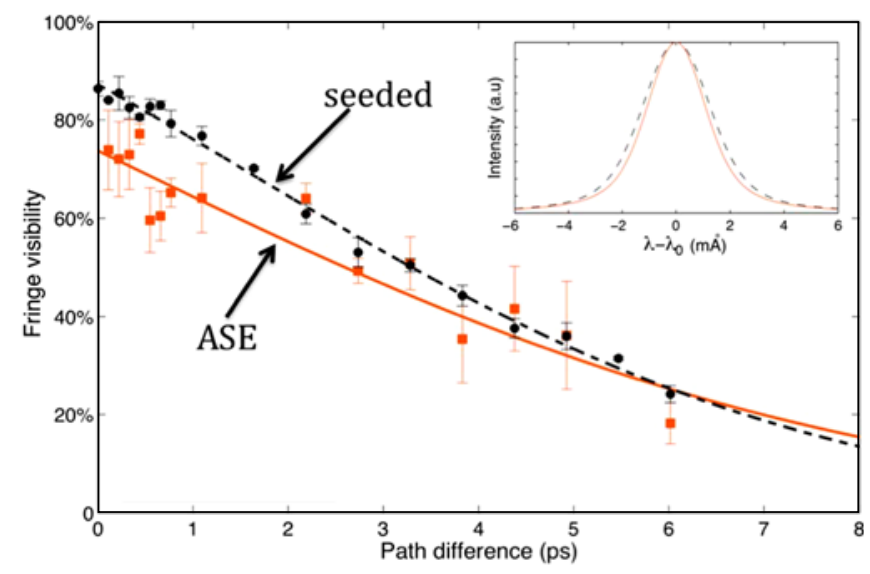

Figure 2. Evolution de la visibilité des franges en fonction de la différence de marche (ici exprimée en temps) introduite dans l'interféromètre. On compare le mode injecté (seeded) et le mode ASE. Les courbes dans l'encart montre les profils spectraux déduits de ces mesures.

On voit sur la figure 2 que le temps de cohérence $\tau_{\mathrm{C}}$ (défini dans la section 2) déduit des mesures est légèrement plus grand dans le mode $\operatorname{ASE}\left(\tau_{\mathrm{c}}=5.5 \pm 0.3 \mathrm{ps}\right)$ que dans le mode injecté $\left(\tau_{\mathrm{c}}=5.1 \pm 0.2 \mathrm{ps}\right)$. Toutefois cette différence est à la limite de notre barre d'erreurs. La largeur spectrale (FWHM) correspondante, déduite du théorème de Wiener-Khintchine, est donc plus grande dans le cas injecté $\left(\Delta v=8.9 \pm 0.610^{10} \mathrm{~Hz}\right.$ ou $\left.\Delta \lambda=3.2 \pm 0.2 \mathrm{~m}\right)$ que dans le cas ASE $\left(\Delta v=7.5 \pm 0.810^{10} \mathrm{~Hz}\right.$ ou $\Delta \lambda=2.7 \pm 0.3 \mathrm{~m}$ ). Rappelons que la largeur spectrale de l'impulsion harmonique injectée dans le plasma est 100 à 1000 fois plus grande que celle de la raie laser XUV. Les simulations numériques effectuées [6] à l'appui de nos mesures montrent que la largeur spectrale de l'harmonique diminue brutalement au cours de l'amplification, mais reste légèrement supérieure. L'effet de rétrécissement de la largeur spectrale de l'harmonique au cours de son amplification a pu être observée dans notre expérience, pour des longueurs de plasma comprises entre 1 et $6 \mathrm{~mm}$ [11].

\subsection{Laser XUV transitoire en mode injecté}

Plus récemment nous avons mesuré la cohérence temporelle du laser XUV transitoire en mode injecté, développé au NSF Center for EUV Science and Technology (Colorado State University, USA). Ce 
laser est émis à 18,9 nm, sur la raie 4d-4p du molybdène nickeloïde, par un plasma produit en irradiant une cible solide de longueur $4 \mathrm{~mm}$ avec une succession de deux impulsions, la deuxième arrivant sous une incidence de $20^{\circ}$ (géométrie GRIP : GRazing Incidence Pumping). Le plasma amplificateur était injecté avec une impulsion harmonique à la même longueur d'onde [12]. La figure 3 montre la courbe de visibilité obtenue. Pour cette mesure nous avons fait varier la différence de marche de part et d'autre du zéro, afin d'améliorer la précision du fit des données autour du maximum de visibilité. Comme précédemment, chaque point résulte d'une moyenne sur 5 à 10 tirs et la barre d'erreur correspond à la déviation standard des fluctuations tir-à-tir. En utilisant la définition du temps de cohérence rappelée plus haut, nous déduisons $\tau_{\mathrm{c}}=1,4 \pm 0,2 \mathrm{ps}$. Cette valeur est donc beaucoup plus petite que celle que nous avons mesurée pour le laser XUV OFI. La courbe de la figure 4 représente le meilleur fit des données, avec une double-exponentielle décroissante. Dans le domaine spectral, ce résultat correspond à un profil Lorentzien de largeur à mi-hauteur $\Delta \lambda=2.7 \pm 0.4 \mathrm{~m}$.

La même mesure a été effectuée en mode ASE et confirme le résultat déjà observé avec le laser XUV OFI : la largeur spectrale est légèrement plus petite en mode ASE $(\Delta \lambda=2.2 \pm 0.3 \mathrm{~m})$. Par ailleurs nous avons également fait varier la longueur du plasma amplificateur pour étudier la présence de rétrécissement de la raie au cours de l'amplification. Les résultats sont présentés en détail dans une autre contribution à ces actes [3].

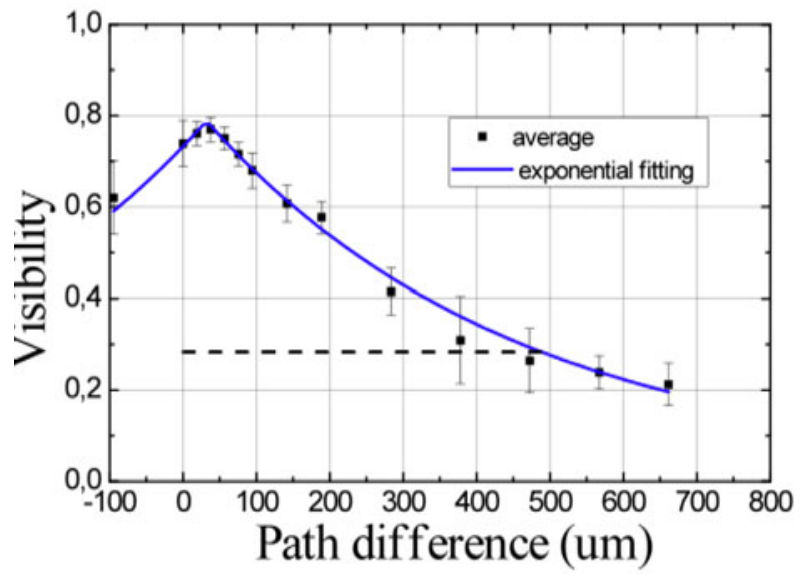

Figure 3. Evolution de la visibilité des franges en fonction de la différence de marche (ici exprimée en longueur) introduite dans l'interféromètre, pour le laser transitoire injecté fonctionnant à 18,9 nm.

Lánalyse détaillée des courbes de visibilité obtenues dans les différentes conditions montre toutefois une difficulté liée à la fluctuation des mesures, qui ne permet pas de trancher précisément entre plusieurs formes fonctionnelles représentant la courbe de visibilité. Or la forme de cette courbe affecte la valeur de la largeur spectrale qu'on en déduit, à travers le facteur numérique qui relie le temps de cohérence $\tau_{\mathrm{c}}$ et la largeur spectrale $\Delta v(\mathrm{FWHM})$. Nous avons donc utilisé une définition différente, basée sur les moyennes quadratiques des grandeurs, et décrite dans [13]. Cette définition permet de s'affranchir de la forme de la courbe de visibilité. Les valeurs obtenues avec cette nouvelle définition sont discutées dans [14]. Elles ne changent pas les principales conclusions de l'expérience, sur l'effet du mode injecté et le rétrécissement de la bande spectrale au cours de l'amplification.

\subsection{Laser XUV à décharge capillaire en mode ASE}

Enfin l'interféromètre a été utilisé pour mesurer la cohérence temporelle du laser XUV émis à 46,9 nm par un plasma d'argon néonoïde généré par une décharge électrique rapide dans un capillaire [15]. Ce 
laser XUV, développé de façon unique dans le laboratoire de JJ Rocca et utilisé pour de nombreuses applications, possède des caractéristiques très différentes des lasers XUV discutés plus haut. En particulier les temps caractéristiques de pompage sont beaucoup plus longs, plusieurs dizaines de nanosecondes au lieu de quelques picosecondes. Il en résulte en particulier une température ionique plus élevée, parce que la thermalisation entre les électrons chauds et les ions a eu le temps de s'effectuer au moment où se créent les inversions de population. D'autre part la densité électronique est ici à nouveau relativement faible, puisque le plasma est produit à partir d'un gaz et non d'une cible solide. On s'attend donc à une contribution plus forte de l'effet Doppler dans les propriétés spectrales de la raie laser XUV.

La figure 4 montre un exemple de courbe de visibilité obtenue, pour un plasma de longueur $33 \mathrm{~cm}$ à une pression d'argon de 160 mTorr. On remarque que les points expérimentaux s'alignent clairement sur une forme Gaussienne, contrairement à ce qui avait été observé avec les lasers XUV pompés par laser décrits plus haut (Figs. 2 et 3). Cette forme Gaussienne pourrait confirmer la dominance de l'effet Doppler dans l'élargissement spectral de la raie. La largeur spectrale mesurée est de $\Delta \lambda=22 \pm 0.7 \mathrm{~m}$, mais le temps de cohérence, $\tau_{\mathrm{c}}=1,8 \pm 0,1$ ps est du même ordre de grandeur que pour le pompage transitoire. L'analyse détaillée et l'interprétation de ces données à l'aide de simulations numériques sont actuellement en cours.

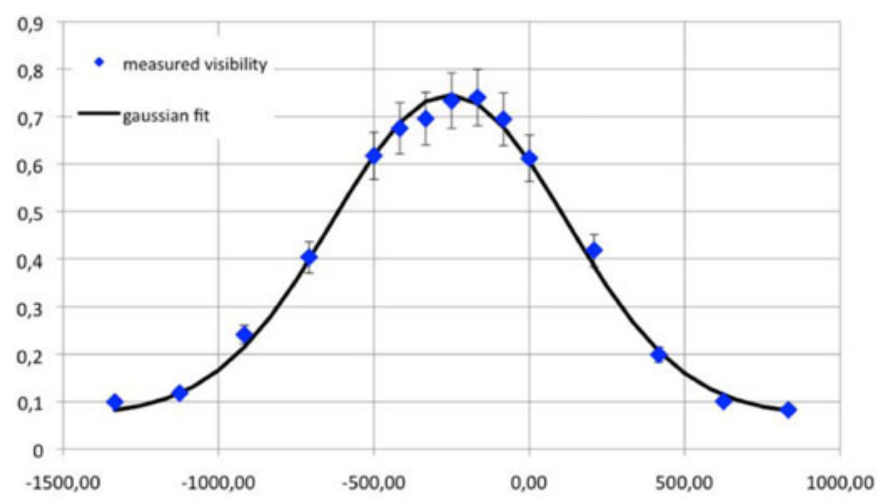

Figure 4. Evolution de la visibilité des franges en fonction de la différence de marche (ici exprimée en microns) introduite dans l'interféromètre, pour le laser à décharge capillaire fonctionnant à $46,9 \mathrm{~nm}$.

\section{CONCLUSION}

Nous avons décrit les résultats d'un ensemble d'expériences qui nous ont permis de caractériser les propriétés spectrales de trois types de lasers XUV collisionnels actuellement les plus avancés. Le but de ce travail est de permettre d'étendre la durée de ces sources au domaine femtoseconde, en élargissant la bande spectrale. Les résultats obtenus indiquent que les lasers XUV transitoires, fonctionnant en mode injectés, sont actuellement les plus prometteurs pour atteindre ce but, avec une durée de cohérence proche de 1 ps. De nouvelles expériences sont prévues pour étudier plus en détail le rôle des paramètres plasmas sur la largeur spectrale, grâce à l'appui des simulations numériques réalisées avec le code PPP [16].

\section{Remerciements}

Les expériences décrites dans cet article ont bénéficié des contrats suivants: LASERLAB Europe, contrat No. 228334 dans le cadre du $7^{\text {ème }}$ Programme Cadre de la CE; NSF Award Number EEC-0310717, et Chemical 
Sciences, Geosciences and Biosciences Division, Office of Basic Energy Sciences, Office of Science, U.S. Department of Energy

\section{Références}

[1] Wang Y et al, Phys. Rev. A 79 (2009) 023810

[2] A. Klisnick et al, J.Q.S.R.T., 99, (2006) 370-380

[3] Meng L. et al, dans ce volume

[4] O. Guilbaud et al, Eur. Phys. J. D., 40, (2006) 125-132

[5] J. Habib et al., Proc. of X-Ray Lasers 2008, Springer Proceedings in Physics, 130 (2009) 115-121

[6] O. Guilbaud et al., Opt. Lett. 35 (2010) 1326

[7] Ph. Zeitoun et al., Nature 431 (2004) 426-429.

[8] J-Ph. Goddet et al., Opt. Lett. 34 (2009) 2438

[9] Y. Wang et al., Phys. Rev. Lett. 97 (2006) 123901

[10] D. Alessi et al., Opt. Lett. 35(2010) 414

[11] F. Tissandier et al., Phys. Rev. A. 81, (2010) 063833

[12] Y. Wang et al., Nat. Phot. 2 (2008) 94

[13] M. Born and E. Wolf, "Principles of Optics" (1980) 6th edition, Pergamon Press, p. 540

[14] L. Meng. et al, submitted to Opt. Lett. (2010)

[15] R. Benware et al., Phys. Rev. Lett. 81 (1998) 5804

[16] A. Calisti et al., J. Quant. Spectro. Rad. Trans. 51 (1994) 59 